\title{
Light clusters in nuclear matter: Excluded volume versus quantum many-body approaches
}

\author{
Matthias Hempel* \\ Department of Physics, University of Basel, Klingelbergstrasse 82, 4056 Basel, Switzerland \\ Jürgen Schaffner-Bielich ${ }^{\dagger}$ \\ Institut für Theoretische Physik, Ruprecht-Karls-Universität, Philosophenweg 16, 69120 Heidelberg, Germany \\ Stefan Typel ${ }^{\ddagger}$ \\ Excellence Cluster Universe, Technische Universität München, Boltzmannstraße 2, 85748 Garching, Germany \\ GSI Helmholtzzentrum für Schwerionenforschung GmbH, \\ Theorie, Planckstraße 1, 64291 Darmstadt, Germany \\ Gerd Röpke ${ }^{\S}$ \\ Institut für Physik, Universität Rostock, Universitätsplatz 3, 18051 Rostock, Germany
}

\begin{abstract}
The formation of clusters in nuclear matter is investigated, which occurs e.g. in low-energy heavyion collisions or core-collapse supernovae. In astrophysical applications, the excluded volume concept is commonly used for the description of light clusters. Here we compare a phenomenological excluded volume approach to two quantum many-body models, the quantum statistical model and the generalized relativistic mean-field model. All three models contain bound states of nuclei with mass number $A \leq 4$. It is explored to which extent the complex medium effects can be mimicked by the simpler excluded volume model, regarding the chemical composition and thermodynamic variables. Furthermore, the role of heavy nuclei and excited states is investigated by use of the excluded volume model. At temperatures of a few $\mathrm{MeV}$ the excluded volume model gives a poor description of the medium effects on the light clusters, but there the composition is actually dominated by heavy nuclei. At larger temperatures there is a rather good agreement, whereas some smaller differences and model dependencies remain.
\end{abstract}

PACS numbers: 21.65.Mn, 26.50.+x, 21.30.Fe, 25.70.Pq

Keywords: Nuclear matter equation of state, Cluster formation, Supernova simulations, Low-density nuclear matter, Relativistic mean-field model, Nuclear statistical equilibrium, Excluded volume

\section{INTRODUCTION}

Clusters in nuclear matter play an important role in various physical systems. Recently, it was shown that the behavior of the symmetry energy in low-energy heavy-ion collisions can only be explained if the formation of clusters is consistently taken into account [1]. The appearance of nuclear clusters in matter below saturation density leads to an increase of the binding energy of symmetric nuclear matter, so that e.g. the symmetry energy gets a finite value at zero density [2]. Very recently there was another attempt to connect predictions from different equations of state (EOS) with experimental data from heavy-ion collisions [3].

Also in astrophysical environments the formation of clusters, respectively nuclei, plays a crucial role. In the crust of cold neutron stars up to $8 \times 10^{6} \mathrm{~g} / \mathrm{cm}^{3}$ the ground state of matter is given by ${ }^{56} \mathrm{Fe}$ [4], which is the nucleus with the lowest total energy (i.e. including rest masses) per nucleon. In general, in catalyzed cold neutron stars only heavy nuclei appear and the fraction of light nuclei like deuterons or alpha particles is vanishingly small. However, this is not the case for systems at finite temperature. Larger temperatures in general favor the appearance of lighter particles, due to the increased entropy. In core-collapse supernovae the entropy per baryon typically ranges from 1 to $20 k_{B}$ and the temperature ranges from 0 to $50 \mathrm{MeV}^{1}$. In the shock heated matter there is a significant contribution of light nuclei, whereas the fraction of heavy nuclei is negligible.

\footnotetext{
*Electronic address: matthias.hempel@unibas.ch

${ }^{\dagger}$ Electronic address: schaffner-bielich@thphys.uni-heidelberg.de

‡Electronic address: s.typel@gsi.de

$\S$ Electronic address: gerd.roepke@uni-rostock.de

1 Throughout the article we use natural units, i.e. $\hbar=c=k_{B}=1$, where it is appropriate.
} 
For astrophysical simulations of e.g. core-collapse supernovae or neutron star mergers, equation of state tables are needed which cover the large domain in baryon number density $10^{-10} \mathrm{fm}^{-3}<n_{B}<1 \mathrm{fm}^{-3}$, temperature $0<T<100$ $\mathrm{MeV}$, and proton fraction $0<Y_{p}<0.6$ (see e.g. Ref. [5]), with a typical resolution summing up to more than one million grid points. Apart from the non-trivial calculation of such tables, one has to encounter the conceptual challenge of a consistent model for the EOS which is able to describe the formation of nuclear clusters and heavy nuclei at low densities as well as the nuclear interactions of uniform nuclear matter at densities above saturation density, with a smooth transition between the different regimes. Due to these difficulties, at present only a few EOS tables are available which are suitable for use in simulations of core-collapse supernovae. In most simulations, the EOS of Lattimer and Swesty (LS) [6] or of H. Shen et al. (STOS) [7, 8] were used. In these two models, besides unbound nucleons and one representative heavy nucleus, only alpha particles are considered. Furthermore, the dissolution of the alpha particle at high densities is only modeled by an excluded volume mechanism. Recently, two new EOS tables were published by G. Shen et al. [9, 10], in which the interaction of alpha particles with the nucleons is described more realistically by using the virial expansion [11] at low densities. In Ref. [12], Hempel and Schaffner-Bielich (HS) developed a new EOS model and later calculated new EOS tables which have been used in simulations of core-collapse supernovae in Ref. [13]. The EOS of HS is based on a nuclear statistical equilibrium model with interactions of the unbound nucleons and excluded volume effects for the nuclei. Important for our purposes here, it also contains all possible light nuclei.

In the simulations of Ref. [13] it was found, in comparison with the LS and STOS EOS, that the alpha particle only poorly describes the entire distribution of all possible light clusters. Especially deuterons and tritons appear with large abundances in the shock heated matter behind the standing bounce shock and can even surmount the alphaparticle fraction. Similar results were found in the study of Sumiyoshi and Röpke [14] before, where light nuclei were included in a core-collapse supernova simulation based on the quantum statistical model of Röpke [15], but only in post-processing of the simulation data. These two studies, and many other theoretical investigations [2, 16-18], show that additional light nuclei appear with large abundances in supernova matter. There is also experimental evidence for this statement: in the previously mentioned heavy-ion experiment similar conditions as in a core-collapse supernova are obtained, and the experimental results cannot be explained without taking light clusters into consideration.

However, there are no simulations of core-collapse supernovae, which consistently take into account all light clusters in the EOS and their interaction with leptons in weak reactions. There exist some interesting exploratory studies. In Ref. [17] the influence of light nuclei on neutrino-driven supernova outflows was investigated and a significant change in the energy of the emitted antineutrinos was found. As another example, in Ref. [16] it was shown, that mass-three nuclei contribute significantly to the neutrino energy loss for $T \geq 4 \mathrm{MeV}$. In the simulations with the EOS of HS [13], weak reactions on the additional light nuclei were described in a simplified way by treating them as alpha particles. The authors of this study conclude that the presence of additional light nuclei might contribute to the neutrino heating in the early post-bounce phase, whereas in the later evolution the effect on cooling might be more important. Still, the question remains to be answered how important the effect of the light cluster distribution will be on the supernova dynamics.

In the present article we compare the statistical excluded volume model (ExV) of HS [12] with two different quantum many-body models, which also include the bound states of light nuclei, the generalized relativistic mean-field (gRMF) model of Typel et al. [2], and the quantum statistical (QS) model of Röpke [15, 19]. The two quantum many-body models give a detailed description of the medium effects on light nuclei. On the other hand, it is much more demanding to include heavy nuclei in the quantum many-body models than in the excluded volume description. This is necessary if one wants to cover the aforementioned parameter range relevant for core-collapse supernova simulations. So far this has been done only for selected thermodynamic conditions by use of the single nucleus approximation [2]. In this approach the full distribution of heavy nuclei is replaced by a single representative nucleus. For the ExV model, EOS tables are already available online, which include the full distribution of light and heavy nuclei, and cover a broad range in density, temperature, and asymmetry. ${ }^{2}$ Here, we want to judge the reliability of the excluded volume approach which is also used in many other EOS models and astrophysical applications and want to see to what extent it can mimic the medium effects of a more microscopic quantum statistical description. We will discuss results for symmetric nuclear matter at a given temperature $T$. Thus the state of the system is defined by $\left(T, n_{B}, Y_{p}\right)$, with the baryon number density being $n_{B}$ and the total proton fraction $Y_{p}=0.5$. In the comparison of the different models we also want to identify the parameter range where heavy nuclei give the dominant contribution to the composition. Furthermore, we will also address the role of excited states of hot nuclei. The article is structured as follows. In Sec. II we briefly present the excluded volume model, and in Sec. III we present the quantum many-body models. In Sec. IV we will compare the results of the three different approaches, before we conclude in Sec. V.

\footnotetext{
${ }^{2}$ See http://phys-merger.physik.unibas.ch/ hempel/eos.html.
} 


\section{EXCLUDED VOLUME MODEL}

The ExV model consists of an ensemble of nucleons and nuclei in nuclear statistical equilibrium, where interactions between the nucleons and excluded volume corrections for the nuclei are implemented. Here we will only give a brief summary of this model; all details can be found in Ref. [12]. For the unbound interacting nucleons (neutrons and protons) a density-dependent relativistic mean-field (RMF) model [20] is applied. Its Lagrangian is based on the exchange of the isoscalar-scalar $\sigma$, the isoscalar-vector $\omega$ and the isovector-vector $\rho$ mesons between nucleons. The coupling strengths are assumed to be density dependent. The free parameters of the Lagrangian, the masses of the mesons and the parameters of the couplings, have to be determined by fits to experimental data. Here we apply the parameter set DD2 [2] in order to use the same approach for the nucleons as the QS and gRMF model in the comparison of the results. The parametrization is based on the same nuclear input as the parameter set DD [20], but experimental nucleon masses are used. Previous tabulations of the ExV model were based on different RMF parameter sets with nonlinear self-interactions of the mesons.

In the ExV model nuclei are treated as non-relativistic classical particles with Maxwell-Boltzmann statistics. We preferably use experimentally measured masses for the description of nuclei. For that we take the nuclear data from the atomic mass table 2003 from Audi et al. [21] whenever possible. For nuclei with an experimentally unknown mass we use the results of the finite-range droplet model (FRDM) in the form of a nuclear mass table [22]. Nuclei behind the neutron drip line are not considered. Below we will show results for two different cases: first, if only the most important light clusters with mass number $A \leq 4$ are considered, and then, if all nuclei with available binding energies are taken into account.

For the screening of the Coulomb field of the nuclei by the background of electrons we use the most basic approximation: each nucleus with mass number $A$ and charge number $Z$ is placed in the center of a spherical Wigner-Seitz (WS) cell with uniform electron density. This leads to the Coulomb correction of the energy of the nucleus:

$$
E_{A, Z}^{\text {Coul }}=-\frac{3}{5} \frac{Z^{2} \alpha}{R_{A}}\left(\frac{3}{2} x-\frac{1}{2} x^{3}\right), x=\left(\frac{n_{B}}{2 n_{B}^{0}} \frac{A}{Z}\right)^{1 / 3}
$$

where $R_{A}$ is the radius of the nucleus with mass number $A$. Here it is assumed that nuclei are uniformly charged spheres at saturation density $n_{B}^{0}=0.149 \mathrm{fm}^{-3}$, the value of the DD2 parametrization $[2]$, so that $R_{A}=\left(3 A / 4 \pi n_{B}^{0}\right)^{1 / 3}$. More elaborated approaches for the Coulomb energy of a multi-component plasma at finite temperature can, e.g., be found in Refs. [23-25]. However, the Coulomb energy becomes only important for heavy nuclei at low temperatures, which is not the main subject of the present investigation. We will even neglect the Coulomb energy for the cases where we only consider the light nuclei with $A \leq 4$.

To take into account excited states of the nuclei, we use a temperature-dependent degeneracy function, i.e. an internal partition function, $g_{A Z}(T)$. It represents the sum over all excited states of a hot nucleus. We choose the simple semi-empirical expression from Ref. [26]:

$$
g_{A Z}(T)=g_{A Z}^{0}+\frac{0.2}{A^{5 / 3} \mathrm{MeV}} \int_{0}^{E_{\max }} d E^{*} e^{-E^{*} / T} \exp \left(\sqrt{2 a(A) E^{*}}\right), a(A)=\frac{A}{8}\left(1-0.8 A^{-1 / 3}\right) \mathrm{MeV}^{-1}
$$

with $g_{A Z}^{0}$ denoting the spin degeneracy of the ground state. For the maximal excitation energy $E_{\text {max }}$ we choose the binding energy of the nucleus (see Ref. [27]). This means we only consider excited states which are still bound. Later we will show results for two cases: Either we consider only the ground-state spin degeneracy or we apply the temperature-dependent internal partition function. We did not include any experimentally known states, because the coverage is rather incomplete and we preferred to have a uniform description for all nuclei. We checked that the results did not change significantly if only the known excited states were used for the light nuclei.

In our description we distinguish between nuclei and the surrounding interacting nucleons, and we still have to specify how the system is composed of the different particles. Let us denote the number density of neutrons and protons by $n_{n}$ and $n_{p}$, respectively, and of the nucleus $(A, Z)$ by $n_{A, Z}$. Thus here and in the following we require $A \geq 2$. The total baryon number density and the total proton number density are then given by:

$$
\begin{gathered}
n_{B}=n_{n}+n_{p}+\sum_{A, Z} A n_{A, Z} \\
n_{B} Y_{p}=n_{p}+\sum_{A, Z} Z n_{A, Z} .
\end{gathered}
$$

For nuclei we will apply the following phenomenological description: All baryons (nucleons in nuclei or unbound nucleons) are treated as hard spheres with the volume $1 / n_{B}^{0}$ so that the nuclei are uniform hard spheres at saturation 
density of volume $V_{A}=A / n_{B}^{0}$. Next, a nucleus must not overlap with any other baryon (nuclei or unbound nucleons). Thus the volume in which the nuclei can move is not the total volume of the system, but only the volume which is not filled by baryons. With these assumptions the free volume fraction is given by:

$$
\kappa=1-n_{B} / n_{B}^{0} .
$$

For the unbound nucleons we use a different description, because the interactions among them are already included in the RMF model. For unbound nucleons we only assume that they are not allowed to be situated inside of nuclei. Therefore we introduce the volume fraction which is not filled by nuclei, i.e. the filling factor of the nucleons:

$$
\xi=1-\sum_{A, Z} A n_{A, Z} / n_{B}^{0}
$$

Next we introduce the number densities of nucleons outside of nuclei, $n_{n}^{\prime}$ and $n_{p}^{\prime}$, i.e. the number of neutrons and protons, respectively, per volume which is not filled by nuclei. They are related to the densities $n_{n}$ and $n_{p}$ which are given by the number of neutrons and protons, respectively, per total volume by:

$$
\begin{aligned}
n_{n}^{\prime} & =n_{n} / \xi \\
n_{p}^{\prime} & =n_{p} / \xi .
\end{aligned}
$$

With these assumptions one derives the following free-energy density:

$$
f=\sum_{A, Z} f_{A, Z}^{0}\left(T, n_{A, Z}\right)+\sum_{A, Z} n_{A, Z} E_{A, Z}^{\mathrm{Coul}}+\xi f_{\mathrm{nuc}}^{0}\left(T, n_{n}^{\prime}, n_{p}^{\prime}\right)-T \sum_{A, Z} n_{A, Z} \ln (\kappa) .
$$

The local number densities $n_{n}^{\prime}$ and $n_{p}^{\prime}$ set the RMF contribution of the nucleons $f_{\text {nuc }}^{0}$, which is weighted with their filling factor $\xi$. This weighting can be expected, as the free energy is an extensive quantity. The first term in Eq. (8)

$$
f_{A, Z}^{0}=n_{A, Z}\left\{M_{A, Z}-T-T \ln \left[\frac{g_{A, Z}(T)}{n_{A, Z}}\left(\frac{M_{A, Z} T}{2 \pi}\right)^{3 / 2}\right]\right\}
$$

is the ideal gas expression for the free-energy density of the nuclei. The Coulomb free energy of the nuclei appears in addition. The last term in Eq. (8) is the direct contribution from the excluded volume. Because of this term, as long as nuclei are present, the free-energy density goes to infinity when approaching saturation density $(\kappa \rightarrow 0)$. Thus nuclei will always disappear before saturation density is reached. If no nuclei are present, we get the unmodified RMF description, as it should be. The excluded volume correction of the nuclei represents a hard-core repulsion of the nuclei at large densities close to saturation density. Similarly, the modification of the free energy of the unbound nucleons is purely geometric and just describes that the nucleons fill only a fraction of the total volume.

Chemical equilibrium between nuclei and nucleons leads to

$$
n_{A, Z}=\kappa g_{A, Z}(T)\left(\frac{M_{A, Z} T}{2 \pi}\right)^{3 / 2} \exp \left(\frac{(A-Z) \mu_{n}^{0}+Z \mu_{p}^{0}-M_{A, Z}-E_{A, Z}^{\text {Coul }}-p_{\mathrm{nuc}}^{0} V_{A}}{T}\right)
$$

where $V_{A}=A / n_{B}^{0}$ is the volume of the nucleus. $\mu_{n}^{0}$ and $\mu_{p}^{0}$, are the chemical potentials of the neutrons and protons, respectively, and the symbol $p_{\text {nuc }}^{0}$ denotes their summed pressure. These quantities are calculated with the relativistic mean-field model for $n_{n}^{\prime}$ and $n_{p}^{\prime}$. Explicit expressions are given e.g. in Ref. [2]. All thermodynamic quantities can be derived consistently in the standard manner from the free energy; the results are given in Ref. [12]. We note that the presented approach for the excluded volume corrections is thermodynamically fully consistent. It is also in agreement with the work of Yudin [28], who studied excluded volume schemes in a rather general form.

$\mu_{n}^{0}, \mu_{p}^{0}$, and $p_{\text {nuc }}^{0}$ contain the RMF interactions of the nucleons. As they appear in Eq. (10) the interactions of the free nucleons are coupled to the contribution of the nuclei. Nuclei are influenced by the mean field of the nucleons. However, in contrast to the generalized RMF model of Typel et al. [2], which we will present in the next section, the bound nucleons do not contribute to the source term of the meson fields, so nuclei do not give a contribution to the mean field. Furthermore, the mutual counteracting in-medium self-energy and the Pauli blocking shifts of the light clusters do not appear. In the ExV model the Mott effect and the dissolution of clusters at large densities is mimicked only by the excluded volume corrections.

One can use also a different value than saturation density for the nucleon density inside of nuclei. It can be seen as a free parameter of the model which determines the strength of the excluded volume effects. This parameter sets the threshold density above which nuclei cannot exist any more. However, we found that other values do not give an overall better agreement with the quantum models. Thus, here we only consider the most intuitive value $n_{B}^{0}$, i.e. the saturation density of the DD2 parametrization. 


\section{QUANTUM MANY-BODY MODELS}

The generalized relativistic mean-field (gRMF) model has been introduced in Ref. [2]. The same parametrization DD2 is applied as for the ExV model. In the gRMF model, in addition to the nucleons, the light clusters are included as quasiparticles which contribute as sources for the meson fields. Like the nucleons, also the light clusters get a mean-field self-energy leading to a reduced effective mass and medium shifts of the chemical potentials. However, the light clusters are composite particles of nucleons. Thus, at large densities the light clusters do not behave as free quasiparticles, but are influenced by the filled Fermi sea of nucleons. This effect is called Pauli blocking and leads to a shift in the binding energies which cannot be described by the gRMF model itself. It is included as a density-dependent part of the nuclear masses, which is taken from the quantum statistical (QS) model in parameterized form.

The QS model is described in detail in Refs. [15, 19]. It is based on the thermodynamic Green's-function method and uses an effective nucleon-nucleon interaction. Effects of the correlated medium such as Pauli blocking, Bose enhancement, and self-energy are taken into account, leading, e.g., to the merging of bound states with the continuum of scattering states with increasing density (the Mott effect). The nucleon self-energies in the QS model are evaluated with the RMF model, neglecting the effect of cluster formation on the mean fields. Then the medium modifications can be determined, such as the mass shift and the Mott densities, where the clusters get dissolved.

As an example, the alpha particle is considered. From a Green's-function approach, we obtain the in-medium four-particle wave equation

$$
\begin{aligned}
& {\left[E^{\mathrm{qu}}(1)+E^{\mathrm{qu}}(2)+E^{\mathrm{qu}}(3)+E^{\mathrm{qu}}(4)-E_{\alpha}^{\mathrm{qu}}(P)\right] \psi_{\alpha, P}(1,2,3,4)} \\
& +\sum_{p_{1}^{\prime} p_{2}^{\prime}}[1-\tilde{f}(1)-\tilde{f}(2)] V\left(1,2 ; 1^{\prime}, 2^{\prime}\right) \psi_{\alpha, P}\left(1^{\prime}, 2^{\prime}, 3,4\right)+\ldots(\text { permutations })=0
\end{aligned}
$$

where five additional interaction terms obtained from different pairings are not given explicitly. Similar equations hold for $A=2,3$. The single-particle energies $E^{\mathrm{qu}}(1)$ contain the self-energy shifts, given, e.g., by the RMF approach. The Pauli blocking contains the phase-space occupation $\tilde{f}(1)$. If we neglect correlations in the surroundings, the actual phase-space occupation (momentum distribution) is replaced by a Fermi function, with a chemical potential that fits the nucleon densities.

The binding-energy shift of the alpha particle

$$
\Delta E_{\alpha}^{\mathrm{qu}}\left(T, n_{B}, Y_{p}, P\right)=E_{\alpha}^{\mathrm{qu}}(P)-E_{\alpha}^{(0)}=\Delta E_{\alpha}^{\mathrm{SE}}(P)+\Delta E_{\alpha}^{\mathrm{Coulomb}}(P)+\Delta E_{\alpha}^{\mathrm{Pauli}}(P)
$$

with respect to the energy in the vacuum $E_{\alpha}^{(0)}$, contains the self-energy shift due to the single-nucleon quasiparticle energies $\Delta E_{\alpha}^{\mathrm{SE}}$, the Coulomb shift $\Delta E_{\alpha}^{\text {Coulomb }}$, and the Pauli blocking shift $\Delta E_{\alpha}^{\text {Pauli }}$. The Pauli blocking shift is relevant for the decrease of the binding energy of nuclei with increasing density. In general, the total binding-energy shift depends on the center of mass momentum $P$ of the nucleus with respect to the medium.

A parametrization of the shifts of the in-medium binding energies is given in Ref. [2]. We use here the more recent parametrization [19] for the QS calculations. The results are nearly identical with the results of the QS calculation shown in Ref. [2]. To evaluate the composition, in the QS calculation also the continuum contributions have been included. In this way the virial EOS is reproduced in the low-density limit. For the single-nucleon quasiparticle shift, the DD2 parametrization was applied to get the same behavior near saturation density as in the ExV and gRMF calculations.

\section{COMPARISON OF RESULTS}

In the following we will compare the results for the three models ExV, gRMF, and QS, for symmetric nuclear matter at three different temperatures. For this comparison we will first only consider nucleons and light clusters with $A \leq 4$, which are also used in Ref. [2], i.e. neutrons, protons, deuterons ${ }^{2} \mathrm{H}$, tritons ${ }^{3} \mathrm{H}$, helions ${ }^{3} \mathrm{He}$, and alpha particles ${ }^{4} \mathrm{He}$. To investigate the role of heavier clusters, we will then include all available nuclei in the ExV model. Finally we will study the role of excited states, by also considering the internal partition function in ExV as presented in Sec. II.

Figure 1 shows the comparison of the composition for $T=4 \mathrm{MeV}$. Let us first focus on the calculations without heavy nuclei, i.e. the thick lines. The results with heavy nuclei (thin dashed and dotted lines) will be discussed later. We depict the mass fractions $X_{i}=A_{i} n_{i} / n_{B}, i \in\{p, d, h, t, \alpha\}$, of protons, deuterons, helions, tritons, and alphas. We note that the fraction of tritons $X_{t}$ is almost equal to the helion fraction $X_{h}$, because they are isospin partners, and we are investigating symmetric nuclear matter. The only differences arise due to the electron screening of the Coulomb interaction and the mass difference. Similarly, the mass fraction of unbound neutrons (which is not shown) is almost equal to the unbound proton fraction $X_{p}$. Up to $n_{B} \sim 10^{-3} \mathrm{fm}^{-3}$ the predictions of the different models 
$\mathrm{T}=4 \mathrm{MeV}$
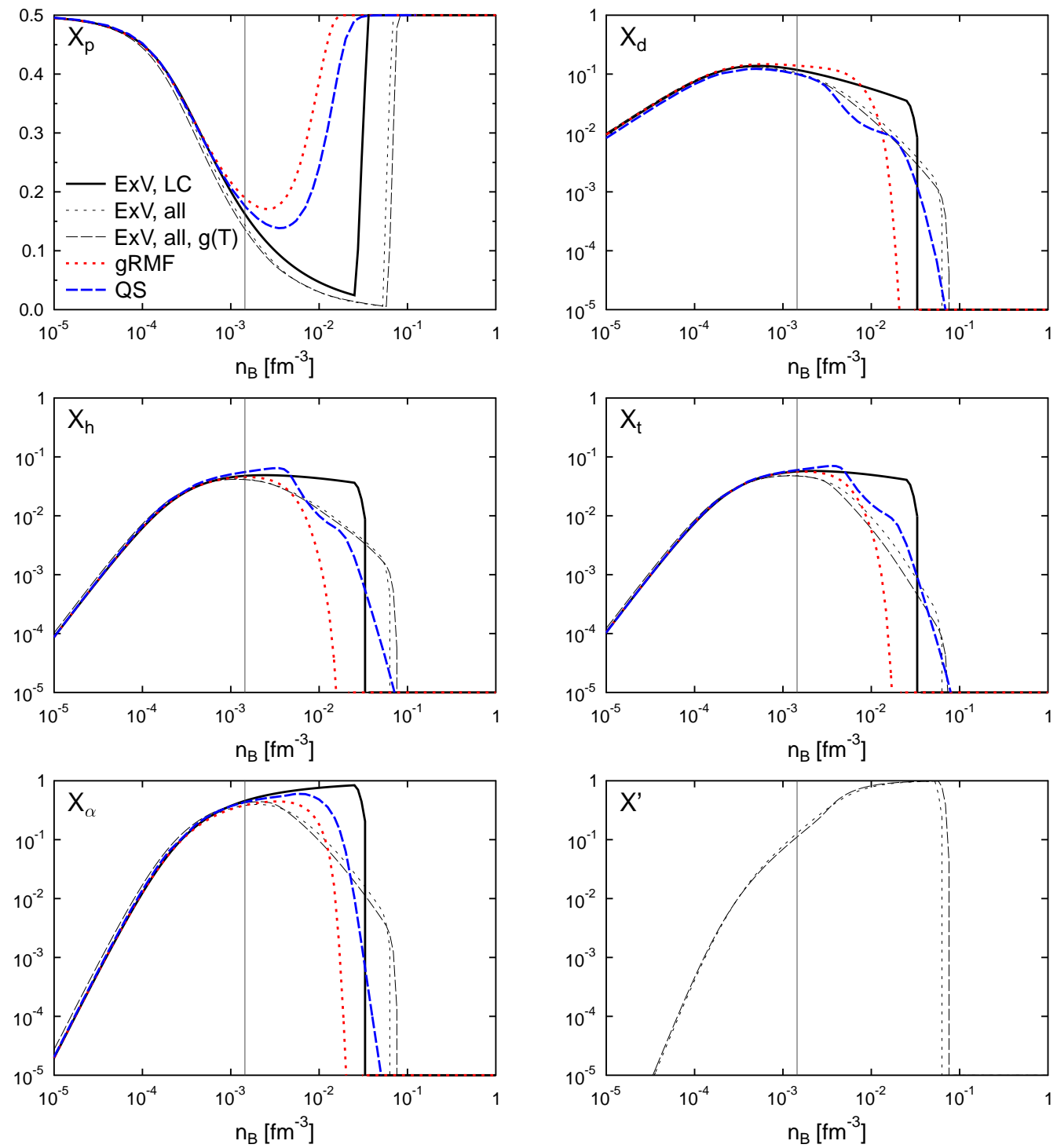

FIG. 1: The mass fractions of protons, deuterons, helions, tritons, and alphas, and the mass fraction $X^{\prime}$, defined by Eq. (13), for symmetric nuclear matter at $T=4 \mathrm{MeV}$. The results of the generalized relativistic mean-field model gRMF and of the quantum statistical model QS from Ref. [2] are compared to the excluded volume model ExV [12]. "ExV, LC" shows the results if only the same light clusters with $A \leq 4$ as in Ref. [2] are considered and no excited states are taken into account. For "ExV, all" all available nuclei are used. "ExV, all, $\mathrm{g}(\mathrm{T})$ " also takes all available nuclei into account, but this time with the internal partition function of excited states. The vertical gray lines show the density where the fraction $X^{\prime}>0.1$ for "ExV, all, $\mathrm{g}(\mathrm{T})$."

for the composition agree with each other. Pronounced differences occur at larger densities. In the QS and gRMF model above the Mott densities, the light clusters start to dissolve due to the Pauli blocking. The binding energies of the light clusters are reduced gradually with density, which leads to an increasing fraction of unbound protons above $n_{B} \sim 5 \times 10^{-3} \mathrm{fm}^{-3}$. Conversely, in the ExV model, the proton fraction decreases and the total fraction of light clusters increases until $n_{B} \sim 0.036 \mathrm{fm}^{-3}$, where a sudden turnover in the composition appears. The two quantum many-body models do not show this behavior. They exhibit a more continuous change of the particle fractions and are rather similar. Still they have different features in detail, like e.g. in the QS model the sinous behavior of some of the mass fractions and larger densities at which the clusters disappear.

The excluded volume approach gives a crude representation of the cluster dissolution at this moderate temperature. However, it is enlightening to study the results of the $\mathrm{ExV}$ model if heavy nuclei are taken into account, depicted by the thin black lines, where the dashed lines include excited states and the dotted do not. At this moderate temperature 

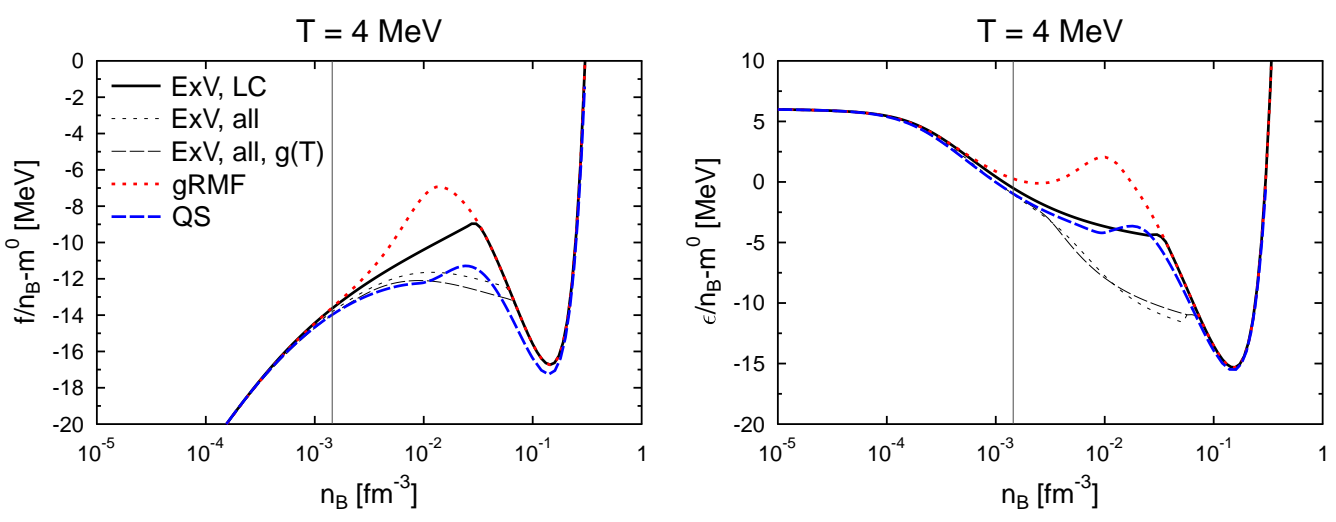

FIG. 2: The free energy per baryon and internal energy per baryon with respect to the averaged rest mass of neutrons and protons at $T=4 \mathrm{MeV}$. Otherwise, notation is as in Fig. 1.

excited states have only a small impact on the composition. In the lower right panel of Fig. 1 we show the sum of the mass fraction

$$
X^{\prime}=1-X_{n}-X_{p}-X_{d}-X_{h}-X_{t}-X_{\alpha}
$$

of all nuclei which are not included in the quantum many-body models (i.e. heavy nuclei with $A>4$ ), but in the ExV model with binding energies using the data base of Audi et al. [21] and the FRDM mass table [22]. One sees that light clusters are actually replaced by heavy nuclei already at intermediate densities. $X^{\prime}$ exceeds 0.1 for densities larger than $1.4 \times 10^{-3} \mathrm{fm}^{-3}$, shown by the vertical lines in Figs. 1 and 2. $X^{\prime}$ gets even larger than 0.9 for densities above $0.016 \mathrm{fm}^{-3}$. Thus the differences which are seen at such large densities in the comparison for $A \leq 4$ are not very significant, because the composition is dominated by heavy nuclei there. The fraction of light clusters is reduced considerably by the appearance of heavy nuclei, before the Mott densities are reached.

For helions and tritons the heavy nuclei lead to an interesting effect. At low densities, where no heavy nuclei exist, the triton fraction is larger than the the helion fraction because of the larger binding energy. However, at densities larger than $\sim 10^{-3} \mathrm{fm}^{-3}$, when the heavy nuclei appear, the triton fraction decreases faster than the helion fraction. Heavy nuclei tend to be asymmetric because of the Coulomb energy. When they give the main contribution to the composition, it is favorable to absorb neutrons from light clusters. We note that the same effect occurs for the unbound neutrons, which is reduced compared to the unbound proton fraction.

In Fig. 2 we show the free energy per baryon $f / n_{B}$ and the internal energy per baryon $\epsilon / n_{B}$ for the different calculations. We subtract the rest mass $m^{0}=1 / 2\left(m_{n}+m_{p}\right)$, corresponding to a proton fraction of 0.5 . The freeenergy density $f$ of the ExV model is specified in Eq. (8), and the internal energy density $\epsilon$ is given in Ref. [12]. The corresponding detailed expressions for the quantities of the quantum many-body models can be found in Ref. [2]. First we discuss the results of Fig. 2, for which only light nuclei are taken into account. Up to the density of $n_{B} \sim 10^{-3}$ $\mathrm{fm}^{-3}$, where the composition of the three different models still agrees, also the displayed thermodynamic variables behave still similarly. At larger densities, even though there is a better agreement for the composition between the QS and the gRMF model, the internal energy of QS is more similar to the ExV model. One reason for the difference between the QS and gRMF models is that the back reaction of the change in the binding energies on the mean fields, mediated by additional rearrangement contributions, is considered in the latter approach. In the QS calculation, the self-energy shifts are taken from the RMF calculation in a parametrized form for pure neutron-proton matter. The free energy of the ExV model without heavy nuclei remains between the two quantum many-body models. One can conclude, that a similar behavior of the composition does not imply in general that other thermodynamic quantities also behave similarly.

The internal energy and free energy of the ExV model change significantly, if heavy nuclei are included, whereas excited states play only a minor role. Deviations due to heavy nuclei appear above $n_{B} \sim 10^{-3} \mathrm{fm}^{-3}$ and become as large as the differences between the QS and gRMF models. It is expected, that also the predictions of the QS and gRMF models for the internal and free energies will be modified considerably if heavy nuclei are taken into consideration in these approaches. However, if we restrict the comparison of the three models to densities below the value shown by the vertical lines, there is an excellent agreement of all three models. When uniform nuclear matter is reached, the three EOSs give identical results, as they are built from the same RMF parametrization DD2.

Figure 3 shows the composition of matter for the three models at $T=10 \mathrm{MeV}$. Again, we first discuss the results where only light nuclei with $A \leq 4$ are considered. There is no sudden turnover of the composition in the ExV 


\section{$\mathrm{T}=10 \mathrm{MeV}$}
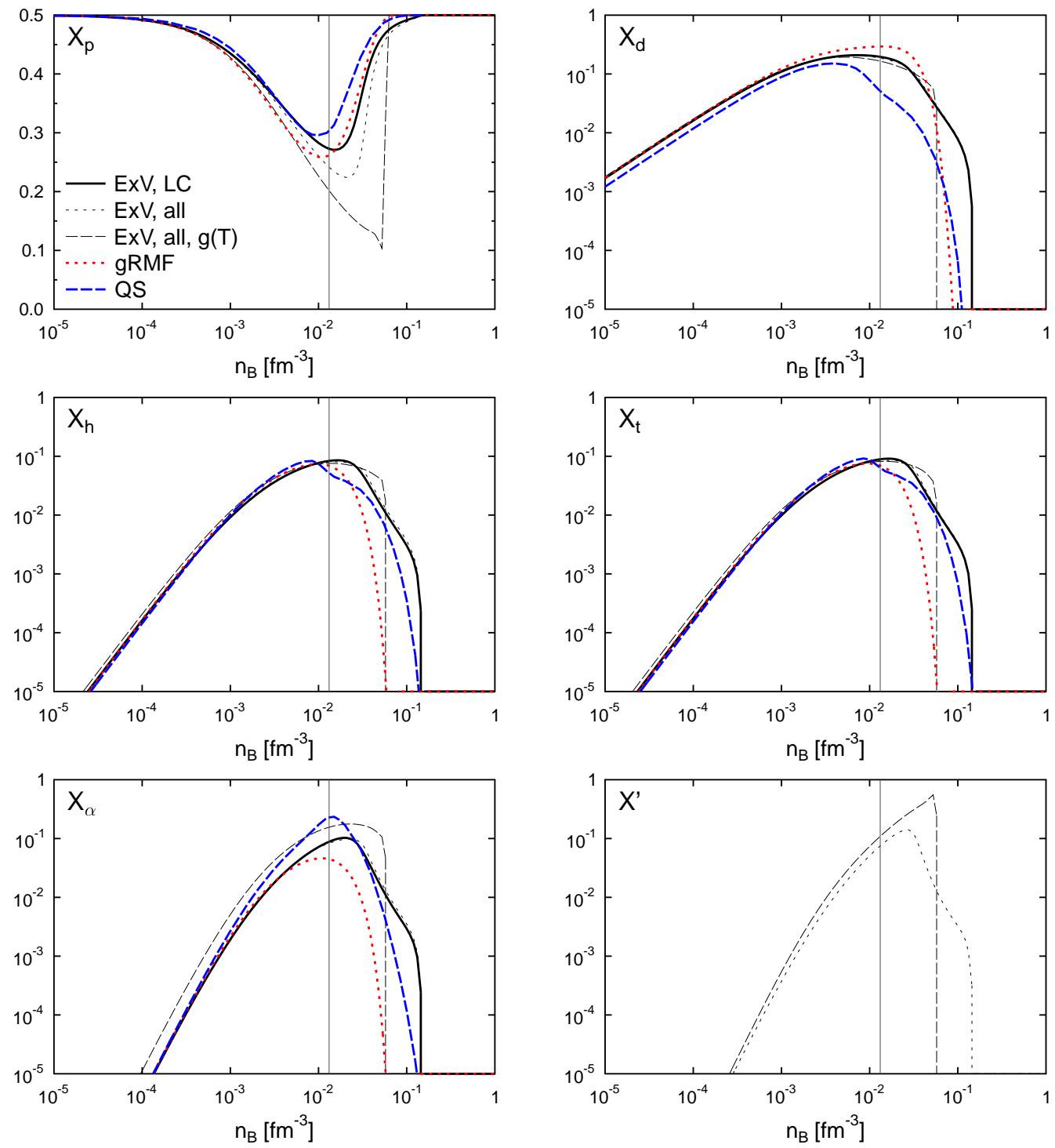

FIG. 3: As in Fig. 1, but now for $T=10 \mathrm{MeV}$.

model any more, but the clusters dissolve in a continuous way. At this temperature the composition of the ExV model agrees much better with the two quantum models. The maximum deuteron and alpha-particle fractions lie between the results of gRMF and QS, and the maximum helion and triton fractions are almost the same as in the QS model. The total cluster fraction of ExV is always close to the two other models, as can be seen from the proton fraction. The densities at which the light clusters disappear completely are slightly larger in the ExV model. In Fig. 3 one sees clearly that the deuteron fraction of QS is slightly reduced, even at very low densities. This is due to the more elaborated treatment of the deuterons in the QS model, in which its continuum contributions are correctly subtracted. Overall the differences of the two quantum models are of similar sizez as the differences to the ExV model. Thus we can conclude that the ExV model mimics the quantum medium effects reasonably well at $T=10 \mathrm{MeV}$. We explain the better agreement at large temperatures by the following aspects. First, the unbound nucleon density is in general larger at larger temperatures, and clusters appear with lower fractions. This is a trivial reason for the closer similarity. Second, the excluded volume corrections give a contribution to the free-energy density proportional to $T \ln (\kappa)$ [see Eq. (8)]. Thus the excluded volume has a larger effect at larger temperatures. On the other hand, also the Pauli-blocking gets weaker at larger temperatures.

The free energy and the internal energy of the models at $T=10 \mathrm{MeV}$ are compared in Fig. 4 . One sees that the ExV model with only light clusters is now closer to the gRMF than to the QS model. However, compared to both 

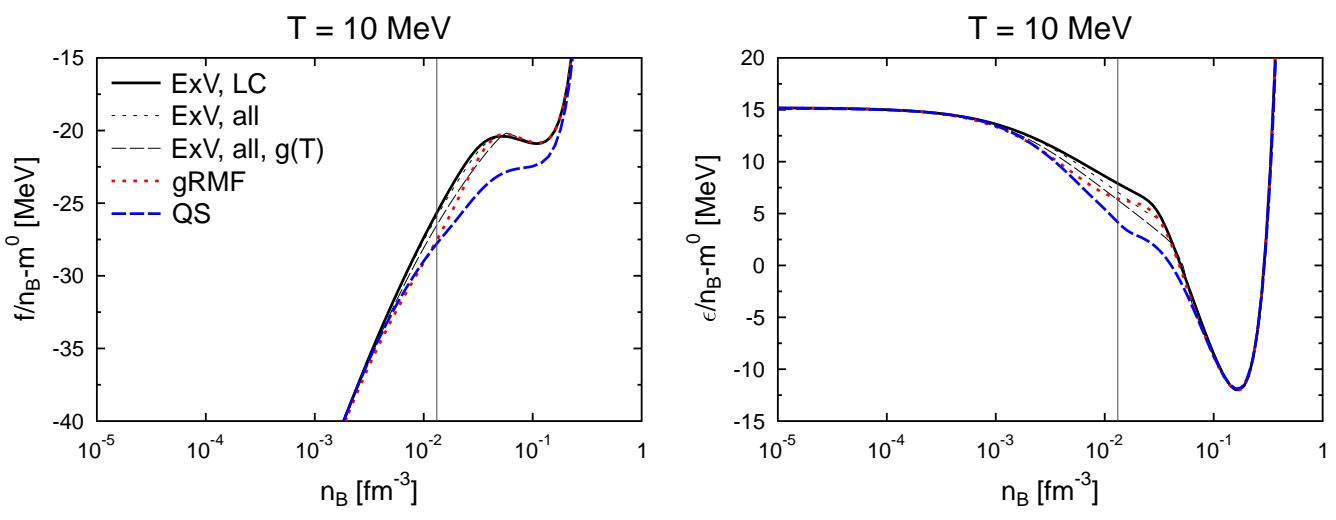

FIG. 4: As in Fig. 2, but now for $T=10 \mathrm{MeV}$.

of the two models, the free and internal energies are increased in the ExV model at almost all densities, even though the composition is similar. As noted before, the contribution to the free energy which originates directly from the excluded volume is proportional to $T$ and leads to an increase of the free energy. However, the excluded volume does not give a direct contribution to the energy density, which is also increased. We have a possible explanation for the observed differences. Regarding the mean field, there are important conceptual differences in the three models: In QS and gRMF all nucleons (bound in clusters and unbound) contribute as sources for the meson fields. Furthermore, the light clusters are treated as quasi-particles and acquire a mean-field self-energy. These effects are absent in the ExV model, where the mean field is given only by the unbound nucleons because the interacting nucleons are assumed to be outside of the light clusters. There is no attraction acting on nuclei, but only the hard-core repulsion.

In the ExV calculation with all nuclei but without excited states (dotted thin black lines in Figs. 3 and 4), one finds that the heavy nuclei for $T=10 \mathrm{MeV}$ are not as important as for $T=4 \mathrm{MeV}$ and that they appear at larger densities. The maximum fractions of the light clusters are reduced only slightly and the transition density to pure nucleon matter remains similar. Here the tritons and helions behave similarly, and the effect of the heavy nuclei observed in Fig. 1 is absent. At $T=10 \mathrm{MeV}$ the inclusion of excited states (dashed thin black lines in Figs. 3 and 4) has a noticeable effect on the EOS and the composition. The formation of heavy nuclei is statistically favored because of the large number of accessible internal states. Their mass fraction exceeds 0.1 at $n_{B} \sim 0.013 \mathrm{fm}^{-3}$ and the maximum mass fraction is $\sim 0.55$ at $n_{B} \sim 0.052 \mathrm{fm}^{-3}$. The internal partition function acts differently on the abundances of the light clusters as can be seen by comparing the dotted with the dashed thin black line in Fig. 3: the alpha-particle fraction is increased most and the deuterons, on the other hand, show no visible change. The deuteron is only very weakly bound with no bound excited state. Thus the internal partition function remains small, in contrast to the much more strongly bound alpha particle with a high cutoff for the maximum excitation energy. This increase of the alpha-particle fraction is also present at very low densities. These results might appear surprising, as the first excited state of the alpha particle lies above $20 \mathrm{MeV}$. However, we also calculated the internal partition function of the alpha particle using only the known excited states and found that the expression of Eq. (2) even underestimates the role of excited states of the alpha particle for the temperatures investigated in this article. In total there are 15 excited states between 20 and $30 \mathrm{MeV}$, with spins of 0,1 , or 2 [29], summing up to a notable contribution.

Similar as for $T=4 \mathrm{MeV}$, the different models give different results for densities larger than $\sim 10^{-3} \mathrm{fm}^{-3}$. Even if we restrict the comparison of the three models with only light nuclei at $T=10 \mathrm{MeV}$ to densities to the left of the vertical lines, where heavy nuclei are not dominant, we still observe some model dependency. Heavy nuclei appear at larger densities than at $T=4 \mathrm{MeV}$, so that the different predictions for light clusters of the three models become relevant. For example, as depicted in Fig. 4, the free energy per baryon differs up to a few $\mathrm{MeV}$, and the internal energy per baryon differs up to $5 \mathrm{MeV}$. The mass fractions of alphas and deuterons can be almost one order of magnitude different, when reaching the vertical line.

For $T=20 \mathrm{MeV}$, which is shown in Fig. 5, the composition of the ExV model evolves similarly with the density when compared with the results of the gRMF and QS models. The maximum mass fractions and the densities at which the single clusters disappear are in a similar range. This supports the conclusion about the effects of temperature which we have drawn before. The reduced deuteron fraction in the QS model, in particular at low densities, is now even more pronounced. As mentioned before, it is due to the more elaborated treatment of the continuum states. The gRMF model, however, shows an increased deuteron fraction as compared to the other approaches at higher densities before the deuteron dissolves. Correspondingly, the proton fraction is reduced. There are important differences in the EOS, which is shown in Fig. 6, for densities where light clusters appear in large concentrations. All three models 


\section{$\mathrm{T}=20 \mathrm{MeV}$}
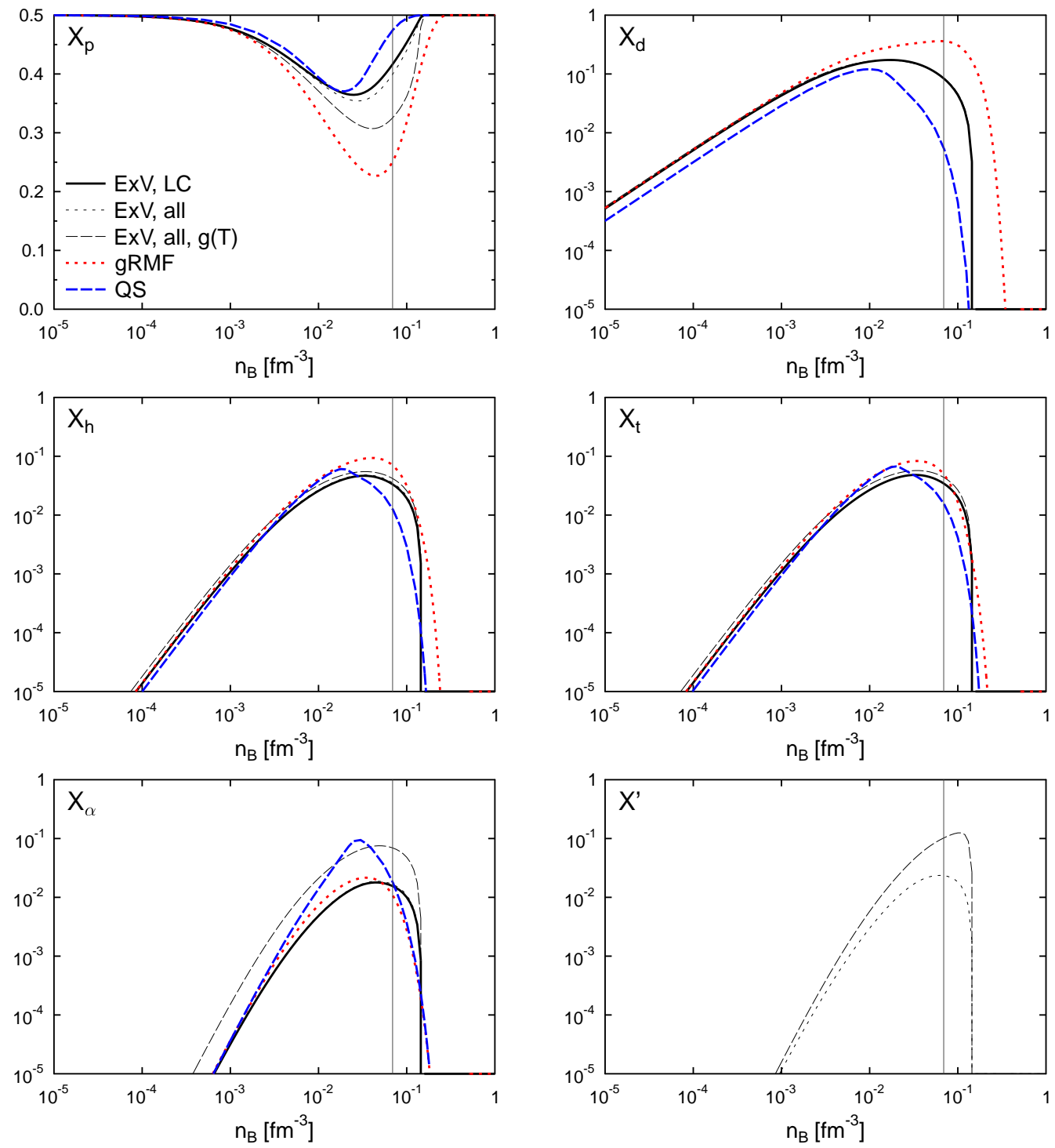

FIG. 5: As in Fig. 1, but now for $T=20 \mathrm{MeV}$.

exhibit rather different behaviors of the energies per nucleon. The ExV model gives the largest energy and free energy, as for $T=10 \mathrm{MeV}$. One also finds that the QS model gives a significantly reduced internal energy compared to the other two models close to saturation density. Fig. 5 shows that heavy nuclei play only a limited role at $T=20 \mathrm{MeV}$, as there are only small differences between the solid and the dotted black lines. If internal excitations of the nuclei are considered in the partition function (dashed black lines) the mass fraction of heavy nuclei increases above 0.1 at $n_{B} \sim 0.069 \mathrm{fm}^{-3}$, but only in a narrow density region. Again, the alpha particles profit the most from the inclusion of the excited states, as their cutoff energy is very large. We remark that the direct contribution of the internal partition function to the EOS (e.g., to the internal energy) is rather small. This is not the case, if no cutoff in the integral for the excited states was used: then arbitrary large excitation energies would give a contribution to the energy density, which would therefore increase with increasing temperature to unphysically large values.

Restricting the comparison of the light cluster models for $T=20 \mathrm{MeV}$ to the left of the vertical lines, there are pronounced differences for the three models, due to the large densities involved. In the composition, the largest differences are found between gRMF and QS. For the shown thermodynamic EOS variables, gRMF and ExV give the most different results, deviating from each other up to $10 \mathrm{MeV}$ for the free energy and $5 \mathrm{MeV}$ for the internal energy. 

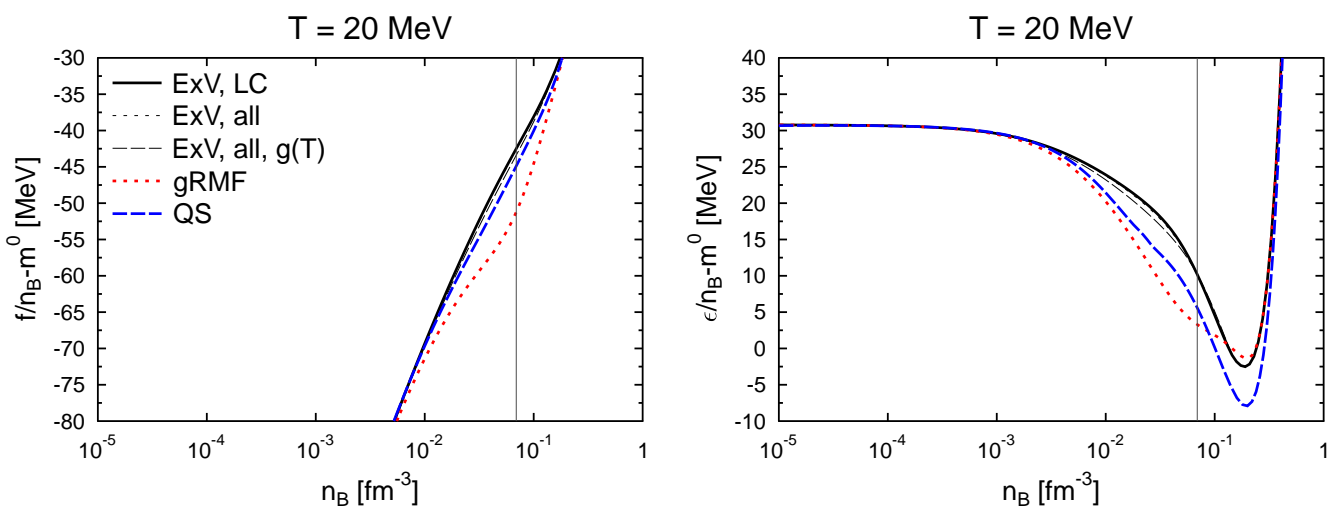

FIG. 6: As in Fig. 2, but now for $T=20 \mathrm{MeV}$.

\section{CONCLUSIONS}

To include cluster formation in the nuclear matter equation of state, medium effects have to be considered that suppress the abundances of light nuclei when the baryon density approaches the saturation value. Basically, within a quantum statistical approach, Pauli blocking as the consequence of anti-symmetrization of fermionic wave functions is responsible for this suppression. Also well known from atomic physics, the Pauli principle between fermions can be considered as repulsion that is depicted by the excluded volume concept. The excluded volume is an empirical parameter that, in simplest approximation, does not depend on other parameter values such as density or temperature. This concept is well proven to derive thermodynamic properties like the Van der Waals EOS. We investigate the inclusion of medium effects to derive the nuclear matter EOS with cluster formation by considering three different approaches: the excluded volume $(\mathrm{ExV})$ approach, the quantum statistical approach based on many-particle theory, and the more challenging generalized relativistic mean-field approach which starts from an effective Lagrangian, but superimposes cluster formation as a first step in a semi-empirical way.

For the composition of dense matter with only light clusters, we conclude that the excluded volume description can imitate the complicated quantum medium effects relatively well at high temperatures. Similar mass fractions are reached and the dissolution of light clusters happens at similar densities as in the two quantum many-body approaches. In contrast, at low temperatures the ExV model behaves very similar to an ideal gas and thus shows crucial deviations. The better agreement at larger temperatures could partly be due to the excluded volume term in the free-energy density, which is proportional to $T$ [see Eq. (8)], and the reduced Pauli blocking. However, if one also considers the results from the ExV model with heavy nuclei, one arrives at partly different conclusions. If the comparison of the three light cluster models is restricted to densities where the mass fraction of heavy nuclei is below 0.1 , the three models agree very well at low temperatures. The previously mentioned crucial deviations of the ExV model at low temperatures are not relevant, because they occur at densities where the composition is actually dominated by heavy nuclei. For larger temperatures heavy nuclei appear at larger densities, so that the slightly different model predictions for light clusters are relevant for the full composition. Further uncertainty may come from the inclusion of excited states which were seen to have a notable effect at large temperatures.

For the EOS, i.e. the internal and free energy per baryon which were investigated here, we found that in general it is not possible to correlate thermodynamic variables like the energy density directly with the composition. Even if two different models show a very similar density dependence of the composition, the EOS can be notably different. For example, there seems to be a systematic over-prediction of the internal and free energy with the ExV model despite the good agreement for the mass fractions with the quantum many-body models at large temperatures. We explained the increased energies by the missing mean-field contribution of light nuclei in the ExV model. The free energy per baryon and internal energy per baryon, respectively, differ for the three models by up to 10 and $5 \mathrm{MeV}$, respectively, for the temperatures investigated here and in the density region where light clusters and nucleons give the dominant contribution.

Although the overall behavior of the mass fractions of light elements agrees reasonably well for the three approaches investigated here, mass fractions of individual light clusters can differ considerably. The accuracy to determine mass fractions is related to the approximations treating the many-particle problem, which become more intricate near the region where the bound states disappear. Obviously, due to its phenomenological character, the excluded volume concept can always only mimic the true quantum effects. A more sophisticated quantum statistical approach has to reproduce not only the nuclear statistical equilibrium in the low-density limit, but also the virial expansion. Therefore 
the account of the contribution of continuum states, included in the QS calculations, is essential, in particular at higher temperatures. Furthermore, for the calculation of the mean fields instead of the approximation of uncorrelated nucleons, correlations have to be included in a self-consistent way (see [15]). Future progress in many-particle theory would improve the accuracy to determine compositions or, more fundamentally, the spectral function at higher densities.

For a more proper comparison with the ExV approach, the contribution of heavy nuclei needs to be considered in the more sound but also more complicated quantum many-body models. However, the results of the ExV approach for light clusters are in satisfactory agreement with the other models. It is promising to continue the investigations of the role of light clusters in core-collapse supernovae and other dynamical astrophysical environments, mentioned in the introduction, e.g., with one of the three models presented here. We conclude that the excluded volume description could be used for such studies to identify the principle effects of the light clusters, followed up with more elaborated models like the quantum many-body models investigated here.

\section{Acknowledgments}

M.H. acknowledges support from the High Performance and High Productivity Computing (HP2C) project. J.S.B. is supported by the German Research Foundation (DFG) within the framework of the excellence initiative through the Heidelberg Graduate School of Fundamental Physics. This work has been supported by the DFG cluster of excellence "Origin and Structure of the Universe" and by CompStar a research networking program of the European Science Foundation (ESF). M.H. is also grateful for participating in the EuroGENESIS collaborative research program of the ESF and the ENSAR/THEXO project.

[1] J. B. Natowitz, G. Röpke, S. Typel, D. Blaschke, A. Bonasera, K. Hagel, T. Klähn, S. Kowalski, L. Qin, S. Shlomo, et al., Phys. Rev. Lett. 104, 202501 (2010).

[2] S. Typel, G. Röpke, T. Klähn, D. Blaschke, and H. H. Wolter, Phys. Rev. C 81, 015803 (2010).

[3] L. Qin, K. Hagel, R. Wada, J. B. Natowitz, S. Shlomo, A. Bonasera, G. Roepke, S. Typel, Z. Chen, M. Huang, et al., e-print arXiv:1110.3345 (2011).

[4] S. B. Rüster, M. Hempel, and J. Schaffner-Bielich, Phys. Rev. C 73, 035804 (2006).

[5] T. Fischer, I. Sagert, G. Pagliara, M. Hempel, J. Schaffner-Bielich, T. Rauscher, F.-K. Thielemann, R. Käppeli, G. Martínez-Pinedo, and M. Liebendörfer, Astrophys. J. Suppl. Series 194, 39 (2011).

[6] J. M. Lattimer and F. D. Swesty, Nucl. Phys. A 535, 331 (1991).

[7] H. Shen, H. Toki, K. Oyamatsu, and K. Sumiyoshi, Nucl. Phys. A 637, 435 (1998).

[8] H. Shen, H. Toki, K. Oyamatsu, and K. Sumiyoshi, Prog. Theor. Phys. 100, 1013 (1998).

[9] G. Shen, C. J. Horowitz, and S. Teige, Phys. Rev. C 83, 035802 (2011).

[10] G. Shen, C. J. Horowitz, and E. O’Connor, Phys. Rev. C 83, 065808 (2011).

[11] C. J. Horowitz and A. Schwenk, Nucl. Phys. A 776, 55 (2006).

[12] M. Hempel and J. Schaffner-Bielich, Nucl. Phys. A 837, 210 (2010).

[13] M. Hempel, T. Fischer, J. Schaffner-Bielich, and M. Liebendörfer, e-print arXiv:1108.0848 (2011).

[14] K. Sumiyoshi and G. Röpke, Phys. Rev. C 77, 055804 (2008).

[15] G. Röpke, Phys. Rev. C 79, 014002 (2009).

[16] E. O'Connor, D. Gazit, C. J. Horowitz, A. Schwenk, and N. Barnea, Phys. Rev. C 75, 055803 (2007).

[17] A. Arcones, G. Martínez-Pinedo, E. O’Connor, A. Schwenk, H.-T. Janka, C. J. Horowitz, and K. Langanke, Phys. Rev. C 78, 015806 (2008).

[18] S. Heckel, P. P. Schneider, and A. Sedrakian, Phys. Rev. C 80, 015805 (2009).

[19] G. Röpke, Nucl. Phys. A 867, 66 (2011).

[20] S. Typel, Phys. Rev. C 71, 064301 (2005).

[21] G. Audi, A. H. Wapstra, and C. Thibault, Nucl. Phys. A 729, 337 (2003).

[22] P. Möller, J. R. Nix, and K.-L. Kratz, Atom. Data Nucl. Data Tables 66, 131 (1997).

[23] D. K. Nadyozhin and A. V. Yudin, Astron. L. 31, 271 (2005).

[24] A. Y. Potekhin, G. Chabrier, A. I. Chugunov, H. E. DeWitt, and F. J. Rogers, Phys. Rev. E 80, 047401 (2009).

[25] A. Y. Potekhin and G. Chabrier, Contrib. Plasma Phys. 50, 82 (2010).

[26] G. Fái and J. Randrup, Nucl. Phys. A 381, 557 (1982).

[27] G. Röpke, Wiss. Z. Univ. Rostock 33, 33 (1984).

[28] A. V. Yudin, Astronomy Letters 37, 576 (2011).

[29] D. R. Tilley, H. R. Weller, and G. M. Hale, Nucl. Phys. A 541, 1 (1992). 